On electromyographic examination fibrillation potentials and positive sharp waves were recorded from the abductor hallucis and flexor digitorum brevis muscles. Motor conduction velocity of the right tibial nerve was slow on the right $(38 \mathrm{~m} / \mathrm{s})$ and normal on the left $(55 \mathrm{~m} / \mathrm{s})$ side; sensory nerve conduction velocity of the right sural nerve and $\mathrm{H}$-reflexes bilaterally were normal.

Eleven months later the pain was localised to the outer aspect of the right calf and foot, and the ankle jerk was diminished on the right side. Weakness of the toe flexors was unchanged, Hoffmann-Tinel's sign was positive over the posterior tarsal tunnel and palpation of the popliteal fossa yielded neither a mass nor tenderness. Sensory nerve conduction velocity of the right medial plantar nerve could not be determined. The distal motor latency of the right tibial nerve was prolonged $(7 \cdot 8 \mathrm{~ms})$.

As the findings were not typical of a tarsal tunnel syndrome, surgery was discouraged.

Thirteen months later MRI of the lower leg up to the popliteal fossa showed no abnormalities other than atrophy of the popliteal and posterior tibial muscles. Electromyography yielded equivocal evidence of neurogenic damage to the posterior tibial muscle prompting examination of the proximal tibial and sciatic nerve by $B$ scan ultrasound. Here a cystic formation of
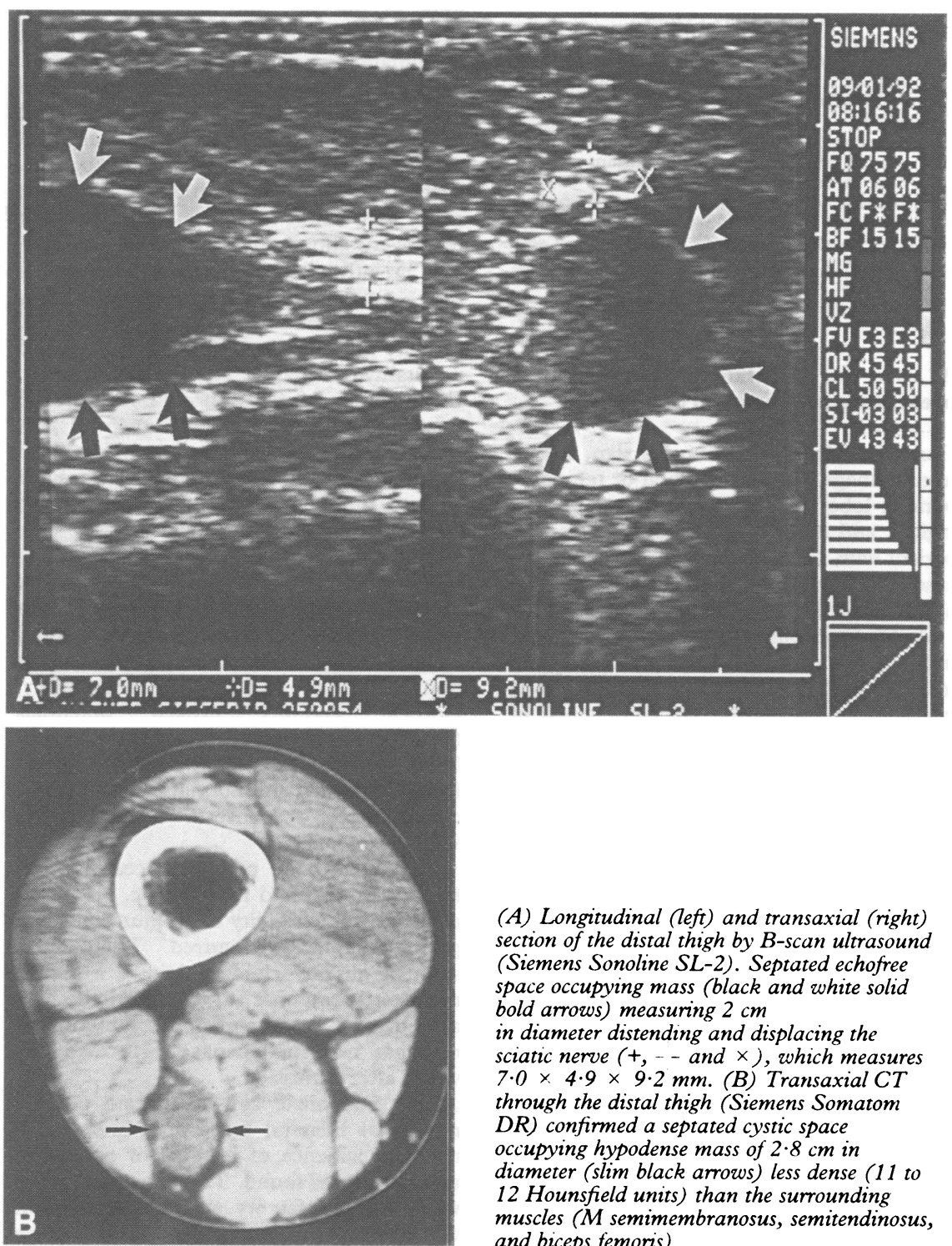

(A) Longitudinal (left) and transaxial (right) section of the distal thigh by $B$-scan ultrasound (Siemens Sonoline SL-2). Septated echofree space occupying mass (black and white solid bold arrows) measuring $2 \mathrm{~cm}$ in diameter distending and displacing the sciatic nerve (,+- - and $\times)$, which measures $7.0 \times 4.9 \times 9.2 \mathrm{~mm}$. (B) Transaxial CT through the distal thigh (Siemens Somatom DR) confirmed a septated cystic space occupying hypodense mass of $2.8 \mathrm{~cm}$ in diameter (slim black arrows) less dense (11 to 12 Hounsfield units) than the surrounding muscles (M semimembranosus, semitendinosus, and biceps femoris). technique which gave the first clue as to the true level of the sciatic ganglion.

Although the ganglion in this patient was seated proximal to the bifurcation of the sciatic nerve into the tibial and peroneal portion, only tibial fibres were affected. The origin of the cyst is not clear. A mucoid degeneration of collagen fibres, metaplasia of irritated connective tissue, ektopia of embryonal synovial tissue, or a mucoid degeneration of a formerly solid tumour for example, a Schwannoma-are possibilities.

After Mahaley's ${ }^{2}$ first report of a ganglion of the (posterior) tibial nerve at the popliteal fossa other ganglia or (Baker's) cysts were also found at the tarsal tunnel. ${ }^{3}$ Only two intraneural synovial cysts of the sciatic nerve were reported between 1980 and $1991 .^{4}$

However rare, a ganglion such as this must be considered in the differential diagnosis of L5 and S1 root, sciatic, tibial and peroneal nerve lesions, as it is amenable to surgery and results are usually favourable. Like other authors ${ }^{5}$ we strongly recommend nerve ultrasonography in any case of progressive peripheral nerve damage of unexplained origin.

The MRI was done by Dr V Buchholz and colleagues, Erlangen.

C J G LANG
The Neurological Hospital

U NEUBAUER

S QAIYUMI

R FAHLBUSCH

The Neurosurgical Hospital

University of Erlangen-Nuremberg

at Erlangen, Germany

Correspondence to: PD Dr Christoph J G Lang, Neurologische Universitätsklinik, Schwabachanlage 6, D-91054 Erlangen, Germany

1 Eiras J, Garcia Cosamalon PJ. Intraneura ganglion of the common peroneal nerve. Neurochirurgia (Stuttg) 1979:22:145-50

2 Mahaley MS Jr. Ganglion of the posterior tib ial nerve. Case report. 7 Neurosurg 1974: 40:120-4.

3 Poppi M, Giuliani G, Pozzati E, Acciarri N, Forti A. Tarsal tunnel syndrome secondary to intraneural ganglion. I Neurol Neurosurg to intraneural ganglion.

4 Gazzeri G, Santucci N. Le cisti sinoviali intraneurali dello sciatico popliteo esterno. Osservazioni su due casi trattati con tecniche microchirurgiche. Minerva Chir 1984 39:1143-5.

5 Leijten FSS, Arts W-F, Puylaert JBCM Ultrasound diagnosis of an intraneural ganglion cyst of the peroneal nerve. $\mathcal{F}$ Neurosurg 1992;76:538-40.

Familial Parkinson's disease and polymorphism at the CYP2D6 locus

Because $10 \%$ of patients with Parkinson's disease have an affected relative ${ }^{1}$ and kindred with a pattern of disease transmission compatible with autosomal dominant inheritance have been described, ${ }^{2}$ genetic factors may play a part in the pathogenesis of the disease. Defective 4-hydroxylation of debrisoquine by CYP2D6, a member of the cytochrome $P-450$ family, has been found in more than $50 \%$ of patients with idiopathic Parkinson's disease, but in less than $20 \%$ of controls. ${ }^{3}$ Four alleles of the CYP2D6 gene, containing point mutations or deletions which inactivate the gene, result in a poor metaboliser phenotype. ${ }^{4}$ The mutant allele CYP2D6B in particular, occurs twice as often in the patients than in the controls, with a relative risk ratio of $2 \cdot 7$. ${ }^{5}$ The risk of idiopathic Parkinson's disease in those with the CYP2D6 mutant 
alleles associated with poor metaboliser phenotype is increased by a factor of $2 \cdot 5 .^{6}$ An alteration in the CYP2D6 gene may therefore also play a part in familial Parkinson's disease.

Segregation analysis was performed in six Parkinson's disease families in which there were at least two affected living patients, with an intragenic microsatellite marker in the CYP2D6 gene. Sixteen patients and 11 relatives were studied. All patients fulfilled the diagnostic criteria for probable Parkinson's disease-namely, (a) at least two of the main features of Parkinson's disease (tremor, rigidity, or bradykinesia), (b) asymmetric onset, $(c)$ pronounced response to levodopa, (d) progressive course, (e) absence of clinical features characteristic of alternative diagnoses or of known aetiology. The mean age at onset was 55 years. DNA was extracted from blood leucocytes, and an intragenic dinucleotide repeat was amplified by the polymerase chain reaction. ${ }^{7}$ Genotypes were determined by hybridisation of a ${ }^{32} \mathrm{P}$ labelled primer to the polymerase chain reaction product after electrophoresis in a denaturing gel and blotting.

Independent segregation of the microsatellite alleles was found in two of the six families (figure). In family 717 , the affected father (II-1) transmitted allele 2 to his affected daughter (III-1) but allele 6 to his affected son (III-2). Although the genotype of the father could only be deduced, false paternity is unlikely, as both children inherited the disease. In family 722, patients III-2 (1/4) and III-10 (2/5) had no marker allele in common, indicating that they do not share an allele of the CYP2D6 gene. These results exclude a defect in this gene as the cause of Parkinson's disease in these two families, unless the disease is not genetic in origin (phenocopy) or there has been a mutation in the dinucleotide repeat. The probability of phenocopy is identical to the prevalence of the disease $(0.35 \%),{ }^{8}$ and the mutation rate for dinucleotide repeats does not exceed $0 \cdot 1 \% .^{9}$ The combined probability for the occurrence of phenocopies and/or microsatellite mutations in both families is therefore $<0.25 \%$. Independent segregation could not be found in the other four families, probably because of their small size.

Although the present study has shown that an allele of CYP2D6 is not responsible for familial Parkinson's disease in two of the six families studied, it cannot be excluded for other cases. Genetic heterogeneity may exist in this disease, as in other neurodegenerative disorders such as Alzheimer's dis- ease and amyotrophic lateral sclerosis. Poor debrisoquine metabolism may also be a risk factor in the pathogenesis of sporadic Parkinson's disease, a question not considered in the present study.

P MAZZETTI E LE GUERN A-M BONNET $M$ VIDAILHET A BRICE Y AGID

INSERM U289 and Fédération de Neurologie, IN Paris Cedex 13, France Present address for P Mazzetti: Instituto Nacional de Ciencias Neurologicas, Lima, Peru

Correspondence to: Professor Yves Agid, Unité INSERM 289, Hôpital de la Salpêtrière, 47 bd de l'Hôpital, 75651 Paris Cedex 13, France.

1 Alonso ME, Otero E, D'Regules R, et al. Parkinson's disease: a genetic study. Can Neurol Sci 1986;13:248-51.

2 Golbe LI, Di Iorio G, Bonavita V, et al. A large kindred with autosomal dominant Parkinson's disease. Ann Neurol 1990;27: 276-82.

3 Barbeau A, Cloutier T, Roy M, et al. Ecogenetics of Parkinson's disease: 4Ecogenetics of Parkinson's disease: 4-
hydroxylation of debrisoquine. Lancet 1985; hydroxylation.

4 Cholerton S, Daly A, Idle J, et al. The role of individual human cytochromes $\mathbf{P 4 5 0}$ in drug metabolism and clinical response. TIPS 1992;13:434-9.

5 Armstrong M, Daly AK, Cholerton S, et al. Mutant debrisoquine hydroxylation genes in Parkinson's disease. Lancet 1992;339. 1017-8.

6 Smith C, Gough A, Leigh N, et al. Debrisoquine hydroxylase gene polymorphism and susceptibility to Parkinson's disease. Lancet 1992;339:1375-7.

7 Polymeropoulos M, Rath D, Xiao H, et al. Dinucleotide repeat polymorphism at the human debrisoquine 4-hydroxylase human debrisoquine 4-hydroxylase 8756.

8 Schoenberg BS, Osuntokin BO, Adeuja AOG, et al. A prevalence survey of Parkinson's disease in the rural United States and in rura Nigeria: door-to-door community studies. Neurology 1988;38:645-6.

9 Weissenbach J, Gyapay G, Dib C, et al. A second generation linkage map of the human genome. Nature 1992;359:794-801.

\section{A case of Creutzfeldt-Jakob disease presenting with cortical deafness}

Cortical deafness is an unusual sign, arising from damage to both temporal or temporoparietal lobes. ${ }^{1}$ The clinical, electrophysiological, and neuropathological findings in a patient with sporadic Creutzfeldt-Jakob disease who presented with cortical deafness are described.

A 65 year old man was admitted for investigation of progressive bilateral hearing loss. It had developed insidiously over two months previously. He complained of

\section{Family 717}

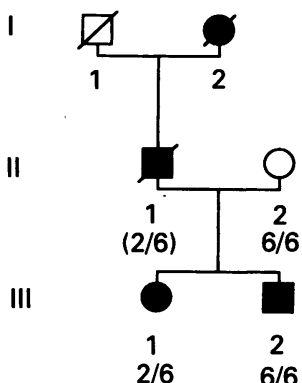

Family 722

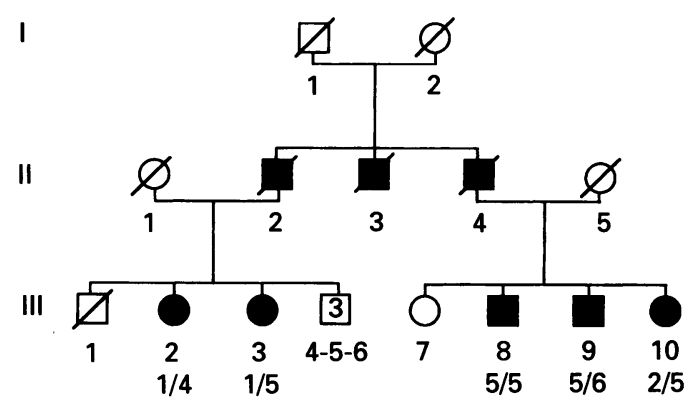

Partial pedigrees of families 717 and 722. Genotypes for CYP2D6 are indicated by large numbers below each tested person. Genotype in parentheses is deduced. "gurgling" noises in his ears, and the loss of hearing could initially be overcome by shouting or increasing the volume of the television. He had difficulty using the telephone. At the time of his admission he could communicate only by writing, and volunteered that his perception of speech was more impaired than his hearing. In the month before admission his wife had noted that he had become more nervous and hesitant. Also during this month, he became unsteady (falling to the left) and developed speech difficulties consisting of hesitancy, stuttering, and a tendency to substitute letters in words ("crudit" instead of "credit").

Rheumatic carditis in childhood had been complicated by mitral valve disease (requiring replacement with a mechanical prosthesis in 1986). A malignant melanoma had been excised in 1991, and a lymph node recurrence treated with excision and radical radiotherapy in 1991. Medication on admission consisted of warfarin, digoxin, Burinex $\mathrm{K}$, and diazepam. There was no family history of deafness or dementia, and no occupational risk factors for cochlear trauma.

Examination showed cachexia but no lymphadenopathy. Severe bilateral hearing loss was confirmed with only non-discriminating perception of sounds. His speech was consistent with deafness (vowel lengthening), but also showed evidence of a more central language disturbance (phonological errors). He had horizontal nystagmus on gaze to the left, and predominantly left sided finger-nose ataxia. The rest of the examination was unremarkable.

The following investigations were normal or negative: routine biochemistry and haematology, erythrocyte sedimentation rate, autoantibody profile, chest radiograph, syphilis serology, international normalised ratio, and serum ammonia. Liver function tests were abnormal (albumin $32 \mathrm{~g} / 1$ (normal 35-50), bilirubin $37 \mu \mathrm{Mol} / 1$ (5-20), alanine aminotransferase $44 \mathrm{U} / 1 \quad(<40)$, aspartate aminotransferase $62 \mathrm{U} / 1 \quad(<40)$, alkaline phosphatase $599 \mathrm{U} / 1$ (70-350) and $\gamma$-glutamyltransferase 222 U/1 $(<50)$ ). Examination of CSF showed less than 5 nucleated cells per $\mathrm{mm}^{3}$ and $0.45 \mathrm{~g} / 1$ protein. Imaging by $C T$ and MRI (with gadolinium) showed no abnormalities. EEG showed prolonged runs of repetitive sharp wave complexes bilaterally. Pure tone audiometry could not be carried out due to lack of cooperation. Brainstem auditory evoked potentials were performed with a suprathreshold $90 \mathrm{~dB}$ click stimulus, and showed waves 3-5 and wave 3 on stimulation of the right and left sides respectively. Cortical auditory evoked potentials (CAEPs) were recorded with an $85 \mathrm{~dB}$ burst stimulus. These showed normal waveforms bilaterally and normal absolute latencies of the $\mathrm{N} 1$ response $(89.5 \mathrm{~ms}$ on right, $99.0 \mathrm{~ms}$ on left).

He deteriorated rapidly, becoming confused, agitated, and more ataxic. He developed paratonic rigidity of all four limbs and primitive reflexes recurred. There were spontaneous myoclonic jerks and a prominent startle response (to touch). He became akinetic and mute, and probably blind (no response to visual menace). He died two weeks after admission.

The immediate cause of death at necropsy was bilateral acute bronchopneumonia. No evidence of residual or metastatic melanoma was found. The prosthetic mitral valve was satisfactory and appreciable left ventricular hypertrophy was noted. The 